\title{
Editorial
}

\section{JunB and PTEN in prostate cancer: 'loss is nothing else than change'}

\author{
P Birner ${ }^{1}$, G Egger $^{1}, 0$ Merkel ${ }^{1,2}$ and L Kenner ${ }^{\star, 1,2,3}$ \\ Cell Death and Differentiation (2015) 22, 522-523; doi:10.1038/cdd.2014.232
}

Prostate cancer (PCa) is the second most common cause of death in men worldwide and $\sim 72000$ die from PCa in the European Union each year (http://seer.cancer.gov/statfacts/html/ prost.html). Owing to its heterogeneous nature, no clear disease pathways have been defined to date, but both genetic and environmental factors appear to be crucial for PCa development. Although most PCas show relatively slow growth and can be controlled by androgen depletion, sometimes for decades, patients may develop a highly aggressive PCa with often fatal outcome. Currently, no suitable prognostic biomarkers are available to distinguish nonaggressive tumors from those that will progress to lethal disease. As a result, patients suffer from substantial and expensive overtreatment with deleterious effects on quality of life such as incontinence and/or impotence. ${ }^{1,2}$

The early stages of prostate carcinogenesis feature alterations in several signaling pathways, the most prominent of which is the $\mathrm{PI} 3 \mathrm{~K}-\mathrm{AKT}$ pathway. In human cancer, $\mathrm{PI} 3 \mathrm{~K}$ is most frequently activated by the inactivation of its negative regulator PTEN. Expression of PTEN inversely correlates with the stage of prostate cancerogenesis, and PCas are often associated with monoallelic PTEN mutations. ${ }^{3}$ Complete loss of PTEN expression is generally associated with metastatic cancers, and several mouse models of PCa are based on conditional deletion of Pten in the prostate epithelium. These mouse models have provided valuable insights into the disease. ${ }^{4}$

PTEN cooperates with a number of signaling pathways, such as the JNK stress kinase pathway, to modulate PCa progression to invasive adenocarcinoma. Thus, PTEN inactivation in human prostate tumors is accompanied by increased JNK activity. ${ }^{5}$ Conversely, JNK ablation in mouse prostate epithelium results in invasive $\mathrm{PCa}$, when combined with PTEN loss. ${ }^{6}$ Thus, JNK signaling via its downstream targets, which includes members of the activating protein 1 (AP-1) family of transcription factors, might have tumor modulating functions. ${ }^{7}$ The AP-1 members c-Fos, C-Jun as well as phosphorylated-cJun are elevated in human PCa samples and lead to the increased proliferation and invasiveness of $\mathrm{PCa}$ cell lines. ${ }^{8}$ Conversely, depletion of JunB in prostatic transient amplifying cells in vitro promoted escape from senescence through the inactivation of $\mathrm{p} 16 / \mathrm{pRb} .^{9}$ This is consistent with the tumorsuppressive functions of JunB in the myeloid and lymphoid lineages. ${ }^{10,11}$
In this issue, Thomsen et al. investigated the role of JunB in the PSA-Cre mediated Pten knockout mouse model, which enables the investigation of the time window from low-grade prostate intraepithelial neoplasia (PIN) to high-grade PIN lesions, which is of special human relevance. ${ }^{12}$ Their results provide yet another in vivo hint as to the tumor-suppressive function of mitogenic signaling mediators. The authors demonstrate that combined knockout of Pten and JunB in prostate epithelial cells results in formation of invasive cancers in vivo. Moreover, the deletion of Pten and JunB by orthotopic delivery of adenoviral Cre recombinase in a subpopulation of adult prostate epithelial cells also resulted in invasive tumor formation. Previous studies showed that PTEN induces the activation of a p53-dependent cellular senescence response. $^{13}$ In the present study, Thomsen et al. observed increased cellular proliferation after the loss of both Pten and JunB and decreased expression of the cell cycle inhibitors p16 ${ }^{\text {Ink4a }}$ and $\mathrm{p} 21^{\mathrm{CIP} 1}$, which are key inducers of cellular senescence (Figure 1). ${ }^{14}$ These findings were supported by data from three different gene expression data sets of human PCa samples, were low-grade tumors express JUNB, but high-grade and metastatic tumors are devoid of JUNB and p21 ${ }^{\mathrm{ClP}}$ expression. Intriguingly, the authors do not observe any similar effect on PIN development by codeletion of Pten and c-Jun, suggesting a specific role for JunB only in limiting PIN progression. It might still be worthwhile to explore the function of $c$-Jun in a more aggressive PCa mouse model such as the $\mathrm{Pb}-\mathrm{Cre}^{15}$

In addition, the authors observed alterations in gene expression in the tumor stroma of Pten; JunB doubleknockout mice, suggesting that the genetic alterations within Pten-deleted tumors can influence the tumor microenvironment. Specifically, the authors describe increased SPP1/ Osteopontin and S100A8/A9 expression in monocytes and macrophages of the tumor stroma derived from doubleknockout mice. This finding is of considerable interest, as increased expression of the SPP1/Osteopontin has been linked to prostate cancerogenesis and metastasis in previous studies. ${ }^{16,17}$ Furthermore, Osteopontin induces a strong potential for intravasation in LNCaP tumor cells. ${ }^{18}$ S100A8/A9 are members of the $\$ 100$ low-molecular weight calcium binding proteins and were shown to be upregulated

\footnotetext{
${ }^{1}$ Department of Pathology, Medical University of Vienna, Vienna, Austria; ${ }^{2}$ Unit of Pathology of Laboratory Animals, University of Veterinary Medicine Vienna, Vienna, Austria and ${ }^{3}$ Ludwig Boltzmann Institute for Cancer Research, Vienna, Austria

${ }^{*}$ Corresponding author: L Kenner, Department of Pathology, Medical University of Vienna, Waehringer Guertel 18-20, 1090 Vienna, Austria. Tel: +43 14040036500 ; Fax: +43 140400 37070; E-mail: lukas.kenner@meduniwien.ac.at
} 


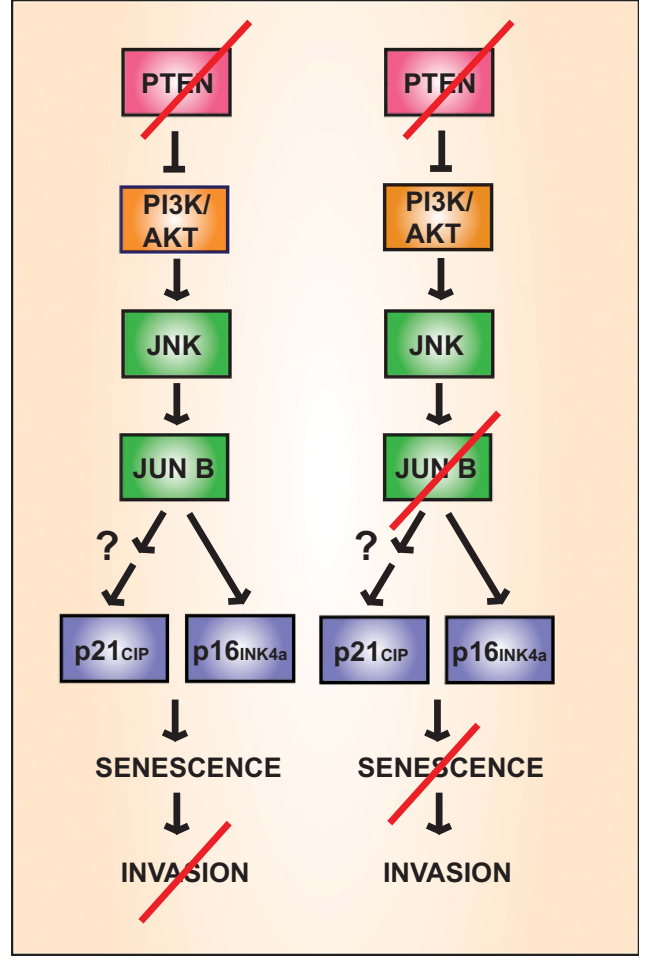

Figure 1 Loss of PTEN and JUNB results in invasive prostate cancer. PTEN deletion is associated with increased PI3K/AKT and mitogenic signaling via JNK and JUNB, which results in oncogene-induced senescence and upregulation of p21 and p16INK4a (left panel). Co-deletion of PTEN and JUNB abrogates senescence, due to downregulation of the cell cycle inhibitors p21 and p16INK4a, and causes invasive prostate cancer

upon loss of JunB in psoriasis. ${ }^{19}$ Increased expression of S100A8/A9 in PCa epithelial cells causes enhanced infiltration of immune cells and stimulates lung-colonization by cancer cells. ${ }^{20}$ The exact mechanism of how the loss of epithelial JunB results in induction of stromal SPP1/Osteopontin and S100A8/A9 expression needs to be further investigated, but possible disruption of this pathway might represent a novel therapeutic opportunity.

Taken together, the present study by Thomsen et al. shows that the combined loss of Pten and JunB has a major impact on the transformation of PIN to invasive PCa, which displays high histological and molecular similarities to human PCa. Although this study adds some fascinating findings to $\mathrm{PCa}$ research, it also generates several new questions as to the diagnostic relevance of JunB loss and to the mechanisms of how JunB and its AP-1 partners influence PCa progression. One very important regulatory mechanism certainly includes the direct transcriptional regulation of $\mathrm{p} 16^{\text {INK4a }}$ by JunB. ${ }^{21}$ However, repression of cell cycle regulators and senescenceassociated genes including $\mathrm{p} 16^{\mathrm{INK} 4 \mathrm{a}}$ has also been associated with epigenetic silencing in diverse tumors. ${ }^{22}$ Two independent silencing mechanisms based on DNA methylation and Polycomb group silencing are both effective in tumors. C-Jun has been shown to protect the promoter of the $\mathrm{p} 16^{\mathrm{INK} 4 \mathrm{a}}$ gene from DNA methylation, whereas the loss of c-Jun evokes DNA methylation of the promoter. ${ }^{23}$ It will be of great interest to investigate how AP-1 can signal to the epigenome and whether direct mechanisms are involved in vivo.
Related to clinical aspects JUNB could provide a novel biomarker for $\mathrm{PCa}$ diagnosis and might help to predict whether a primary tumor has the potential to become invasive and disseminate to distant sites. Thus, mitogenic signaling pathways are central to the regulation of diverse cellular pathways including proliferation and senescence and can have opposing effects on tumorigenesis depending on the stage, by provoking tumor promotion or suppression. We can expect that additional partners of these pathways are important to balance proliferative versus cell cycle inhibitory effects, and an elucidation of the underlying mechanisms will increase our understanding of tumorigenesis.

1. Prensner JR, Rubin MA, Wei JT, Chinnaiyan AM. Beyond PSA: the next generation of prostate cancer biomarkers. Sci Transl Med 2012; 4: 127rv123.

2. Wilt TJ, MacDonald R, Rutks I, Shamliyan TA, Taylor BC, Kane RL. Systematic review: comparative effectiveness and harms of treatments for clinically localized prostate cancer. Ann Intern Med 2008; 148: 435-448.

3. Salmena L, Carracedo A, Pandolfi PP. Tenets of PTEN tumor suppression. Cell 2008; 133 : 403-414.

4. Parisotto M, Metzger D. Genetically engineered mouse models of prostate cancer. Mol Oncol 2013; 7: 190-205.

5. Vivanco I, Palaskas N, Tran C, Finn SP, Getz G, Kennedy NJ et al. Identification of the JNK signaling pathway as a functional target of the tumor suppressor PTEN. Cancer Cell 2007; 11: $555-569$.

6. Hubner A, Mulholland DJ, Standen CL, Karasarides M, Cavanagh-Kyros J, Barrett T et al. JNK and PTEN cooperatively control the development of invasive adenocarcinoma of the prostate. Proc Natl Acad Sci USA 2012; 109: 12046-12051.

7. Davis RJ. Signal transduction by the JNK group of MAP kinases. Cell 2000; 103: 239-252.

8. Edwards J, Krishna NS, Mukherjee R, Bartlett JM. The role of c-Jun and c-Fos expression in androgen-independent prostate cancer. J Pathol 2004; 204: 153-158.

9. Konishi N, Shimada K, Nakamura M, Ishida E, Ota I, Tanaka N et al. Function of JunB in transient amplifying cell senescence and progression of human prostate cancer. Clin Cancer Res 2008; 14: 4408-4416.

10. Passegue E, Jochum W, Schorpp-Kistner M, Mohle-Steinlein U, Wagner EF. Chronic myeloid leukemia with increased granulocyte progenitors in mice lacking junB expression in the myeloid lineage. Cell 2001; 104: 21-32.

11. Laimer D, Dolznig H, Kollmann K, Vesely PW, Schlederer M, Merkel O et al. PDGFR blockade is a rational and effective therapy for NPM-ALK-driven lymphomas. Nat Med 2012; 18: $1699-1704$

12. Ma X, Ziel-van der Made AC, Autar B, van der Korput HA, Vermeij M, van Duijn $P$ et al. Targeted biallelic inactivation of Pten in the mouse prostate leads to prostate cancer accompanied by increased epithelial cell proliferation but not by reduced apoptosis. Cancer Res 2005; 65: 5730-5739.

13. Chen Z, Trotman LC, Shaffer D, Lin HK, Dotan ZA, Niki M et al. Crucial role of p53-dependent cellular senescence in suppression of Pten-deficient tumorigenesis. Nature 2005; 436: 725-730.

14. Larsson LG. Oncogene- and tumor suppressor gene-mediated suppression of cellular senescence. Semin Cancer Biol 2011; 21: 367-376.

15. Wang S, Gao J, Lei $Q$, Rozengurt N, Pritchard C, Jiao J et al. Prostate-specific deletion of the murine Pten tumor suppressor gene leads to metastatic prostate cancer. Cancer Cell 2003; 4: 209-221.

16. Ramankulov A, Lein M, Kristiansen G, Loening SA, Jung K. Plasma osteopontin in comparison with bone markers as indicator of bone metastasis and survival outcome in patients with prostate cancer. Prostate 2007; 67: 330-340.

17. Castellano G, Malaponte G, Mazzarino MC, Figini M, Marchese F, Gangemi P et al. Activation of the osteopontin/matrix metalloproteinase-9 pathway correlates with prostate cancer progression. Clin Cancer Res 2008; 14: 7470-7480.

18. Khodavirdi AC, Song Z, Yang S, Zhong C, Wang S, Wu H et al. Increased expression of osteopontin contributes to the progression of prostate cancer. Cancer research 2006; 66: 883-888.

19. Zenz R, Eferl R, Kenner L, Florin L, Hummerich L, Mehic D et al. Psoriasis-like skin disease and arthritis caused by inducible epidermal deletion of Jun proteins. Nature 2005; 437: 369-375.

20. Grebhardt S, Muller-Decker K, Bestvater F, Hershfinkel M, Mayer D. Impact of S100A8/A9 expression on prostate cancer progression in vitro and in vivo. J Cell Physiol 2014; 229: 661-671.

21. Passegue $E$, Wagner $E F$. JunB suppresses cell proliferation by transcriptional activation of p16(INK4a) expression. The EMBO J 2000; 19: 2969-2979.

22. Zane L, Sharma V, Misteli T. Common features of chromatin in aging and cancer: cause or coincidence? Trends Cell Biol 2014; 24: 686-694.

23. Kollmann K, Heller G, SexI V. c-JUN prevents methylation of p16(INK4a) (and Cdk6): the villain turned bodyguard. Oncotarget 2011; 2: 422-427. 